\title{
Characteristic of CD4+CD25+ T Cells in Chronic Myeloid Leukemia Patients Treated with Imatinib Mesylate with Different BCR-ABL Transcripts Levels Response
}

\author{
Shahla'a Fadhil Sabir ${ }^{1,2}$, Wifaq Mahmood Ali Alwatar ${ }^{3}$, Bassam Francis Matti ${ }^{4,5}$ \\ 1Lecturer, The National Center of Hematology, Al-Mustansiriyah University, Baghdad, Iraq, ${ }^{2}$ Microbiology \& \\ Immunology Department, College of Medicine, University of Baghdad, Baghdad, Iraq, ${ }^{3}$ Assist. Prof. Unit of \\ clinical \& communicable disease, College of Medicine, University of Baghdad, Baghdad, Iraq, ${ }^{4}$ Consultant Adult \\ Clinical Hematology Department, Baghdad Teaching Hospital, Medical City, Baghdad, Iraq, ${ }^{5}$ Bone Marrow \\ Transplant Center, Medical City, Baghdad, Iraq
}

\begin{abstract}
Background: Several clinical trials on cancer showed a correlation between elevated levels of regulatory $\mathrm{T}$ cells with either poor prognosis or poor response to some therapies. Hence, in this study we tried to measure the regulatory $\mathrm{T}$ cells $(\mathrm{CD} 4+\mathrm{CD} 245+)$ count and to evaluate the program death receptor $1(\mathrm{PD} 1)$ as a one of the main suppressive mechanisms that regulatory $\mathrm{T}$ cells use in CML patients with different molecular response to imatinib therapy. Method: Flow cytometry technique was used to analyze 30 sample of optimal molecular response of CML patients (BCR-ABL transcripts $\leq 0.1 \%$ ) and 30 sample of failure molecular response patients (BCR-ABL transcripts $>1 \%$ ) with or without hematological failure, in order to assess the CD4+CD25+ T cells (Tregs) and PD1 expression on these cells. Thirty samples age and gender matched were used as healthy controls. Results: A high proportion of CD4+CD25+ T cells was found in failure groups compared to control and optimal groups $(\mathrm{P}=<0.001)$, while there was no significant difference between control and optimal groups with $(\mathrm{P}=0.481)$. A cutoff value of $\mathrm{CD} 4+\mathrm{CD} 25+\mathrm{T}$ cells at $>33.7 \%$ was highly significant with high sensitivity and specificity. This value can be used to determine the failure from the optimal CML responders. There was no noticeable differences $(\mathrm{P}=0.125)$ in $\mathrm{PD} 1+$ expression among CD4+CD25+ T cells. Conclusion: A high percentage of Treg cells in the failure CML group might be an indication of immune escape of leukemic cells in those patients compared to the other investigated groups.
\end{abstract}

Keywords: CML, Imatinib mesylate, CD4+CD25+ Tregs, PD1.

\section{Introduction}

In hematological malignancies, such as chronic myeloid leukemia (CML), CD8 $+\mathrm{T}$ cells are downregulated in patients at time of diagnosis ${ }^{(1)}$, as the immune system becomes impaired favoring immune escape of the leukemic cells ${ }^{(2)}$. CML is characterized by the presence of BCR-ABL fusion oncogene that encodes BCR-ABL1 oncoproteins that have high tyrosin kinase

\footnotetext{
Corresponding Author: Shahla'a Fadhil Sabir

Microbiology \& Immunology Department, College of Medicine, University of Baghdad, Baghdad, Iraq e-mail: shahlaa_fadhil@yahoo.com
}

activity (3). Imatinib mesylate, a selective tyrosine kinase inhibitor, is considered the first TKI in CML management, accomplishing high response rates and overall survival, nevertheless; some of CML patients still develop treatment failure ${ }^{(3)}$. The TKI treatment responses are more potent in patients with less exhausted $\mathrm{T}_{\text {cells }}{ }^{(4)}$. A subset of CD4+ cells defined as a regulatory $\mathrm{T}$ cells (Tregs), which are reported as $\mathrm{CD} 4+\mathrm{CD} 25+$, express a high level of CD25+ (IL-2Ra), and can exhibit a suppressive function ${ }^{(5)}$.

Tregs control effector immune cells by humoral and cell to cell contact mechanisms ${ }^{(6)}$. Host environment and PD1 signaling play an important role in Treg development ${ }^{(7)}$. In malignancy, Tregs considered a vital suppressive immunocyte in tumor microenvironment 
(TME) and are characterized by PD1 and PDL1 expression, and this demonstrated it contribution to the tumor progression ${ }^{(7)}$.

In the CML treatment era, the main goal is to achieve a durable deep molecular response for treatment-free remission to stope TKI therapy. The immunological response and its effect in responder patients of CML may have a substantial value for those with a lack of overt relapse ${ }^{(8)}$. From other view, the poor immunological response in CML can be part of targeted therapy to induce better response and to control TKI response failure.

Here in this study, we are trying to understand the dynamics of immune escape in CML patients by assessing Treg cells, that express CD4+CD25+ and PD1+ among different molecular response levels of CML patients undergoing imatinib therapy.

\section{Materials and Method}

Subjects: This study was conducted between Nov.2018-Dec.2019 at Baghdad Teaching Hospital/ Medical City and The National Center of Haematology. Sixty patients with CML were enrolled in the present study, age $>18$ years old and on imatinib mesylate therapy for more than 1 year duration. Patients were grouped according to treatment response and the level of BCR-ABL transcripts. Treatment response criteria were defined according to the European Leukemia-Net (ELN) guidelines ${ }^{(9)}$. Thirty patients were classified as an optimal responders (p210 BCR-ABL transcript levels $\leq 0.1 \%$ ) and 30 patients were classified as had a failure molecular response to therapy with or without loss of hematological response (p210 BCR-ABL transcript levels $>1 \%$ ). This study was approved by the scientific ethics committee/faculty of medicine, university of Baghdad. Thirty age and gender matched samples without history of hematological or chronic disease were taken as a control group. At time of sampling, blood count indices were obtained and calculated by automated blood count analyzer.

Monoclonal antibodies and flow cytometry analyses: The flow cytometry analysis was performed on peripheral blood at a maximum interval of 24 hours. Fluorescently labeled monoclonal antibodies (mAbs) included [anti-CD45 (FITC), anti-CD4 (APC/CY7), anti-CD25 (APC) and anti-CD279 (PE/CY7)] purchased from (Biolegand, USA), flow cytometry staining was performed according to Biolegend immunofluorescence staining protocol. Data analyzed with DIVA software version 2016 using an immunological gate (SSC/CD45), along with CD4 marker were used to differentiate CD4+T-lymphocyte in all studied groups. At least 100,000 events were analyzed for each sample.

Statistical analysis: Data analysis was done with SPSS 22.0.0 (Chicago, IL). For comparison between different groups, one-way ANOVA is used, and post Hoc Tukey test is used to confirm which groups are significant. Receiver operator curve (ROC) is used to see the validity of Tregs in recognizing failure cases from optimal, $(\mathrm{P} \leq 0.05)$ were considered statistically significant.

\section{Results}

General characterization of Patients and control group: Out of $60 \mathrm{CML}$ patients on imatinib therapy, 30 patients were with optimal response with (mean age $43.47 \pm 13.14$ years; mean disease duration $73.7 \pm 37.6$ months; $\mathrm{M} ; \mathrm{F}$ ratio $1.72: 1$ ) and 30 patients were with failure response with (mean age 50.13 \pm 13 .61years; mean disease duration $69.4 \pm 46.4$ months; $\mathrm{M}$; F ratio 0.76:1). Thirty healthy volunteer age and gender matched were included and evaluated as control samples with (mean age 46.33 \pm 8.84 years; $\mathrm{M}$; F ratio 1.14:1).

Patient's characteristics according complete blood count were included: Haemoglobin $($ mean \pm SD) levels for the control, optimal response and failure response were $(14.00 \pm 1.68 \mathrm{~g} / \mathrm{dL}, \quad 12.38 \pm 1.59 \mathrm{~g} / \mathrm{dL}$ and $11.11 \pm 2.04 \mathrm{~g} / \mathrm{dL}$, respectively) with a significant difference among studied groups as shown in (Fig. 1A).

Regarding the platelets count (pltx $\left.10^{3} / \mu \mathrm{L}\right)$, the median for the control group was 312, range (247.3322.3), for optimal response was 254.5, range (201.3297.5) and for failure response was 300, range (146.8559.8), with a significant difference only between optimal responder CML and control group as shown in (Fig. 1B).

The median for white blood cells count (WBCx $10^{3 /}$ $\mu \mathrm{L}$ ) for control was 7.63, range (6.65-8.81). For optimal response was 6.0, range (5.15-7.29). For failure response patients was 10.4, range (5.6-27.68), with no significant difference only between control and failure CML response group $(\mathrm{P}=0.999)$ as shown in (Fig. 1C). 
A)

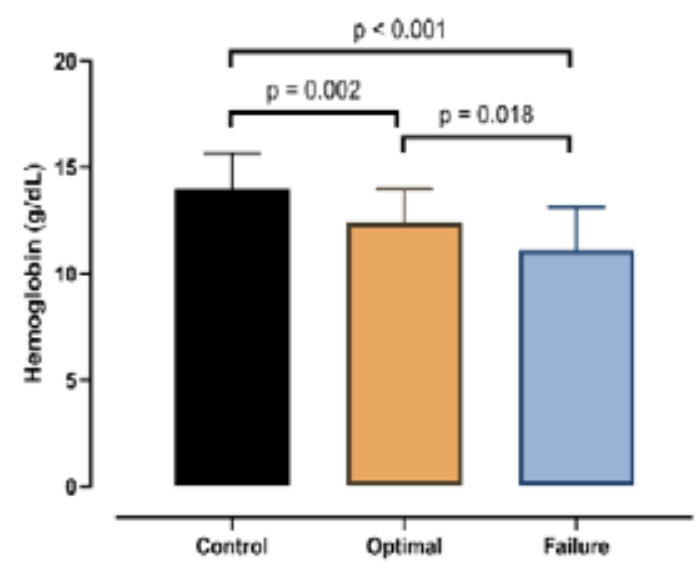

C)

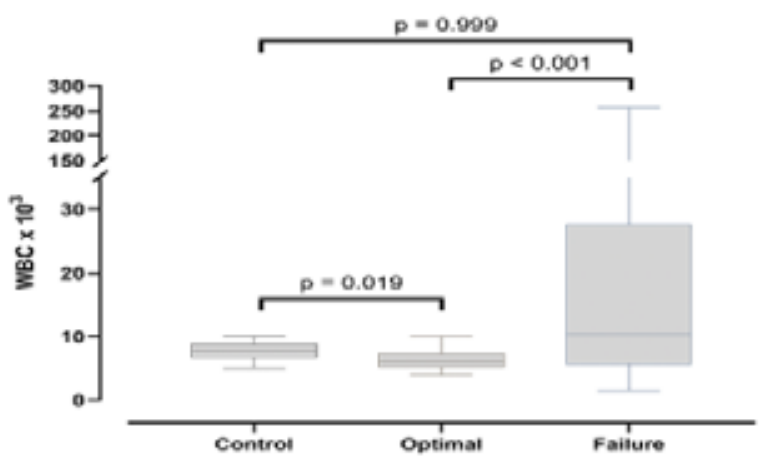

B)

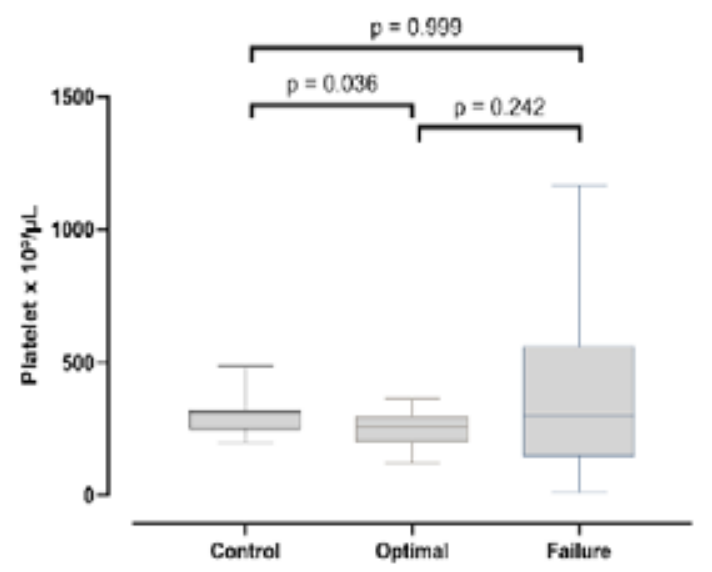

Figure 1: Patients characteristics according complete blood count among different CML groups and control group. A) Hemoglobin levels B) Platelet count C) WBCs count.

All CML cases were categorized according to imatinib therapy response depending on the results of QPCR for p210 BCR-ABL transcripts and peripheral blood indices for each patient. There was a significant difference ( $\mathrm{P}=<0.001)$ between failure response group with mean $(28.4 \pm 25.1 \%)$ and optimal response group with mean $(0.02 \pm 0.03 \%)$ regarding the BCR-ABL transcript levels. All patients with optimal response were with major molecular response (p210 BCR-ABL transcripts $\leq 0.1 \%)$, and $19(63.3 \%)$ of them had molecular response with BCR-ABL level $(0.1->0.0032 \%) \log 3$ \& 4 reduction in BCR-ABL1 transcripts levels, while the other responders patients with 11(36.7\%) had BCR$\mathrm{ABL}(\leq 0.0032) \log 4.5 \& 5$ reduction levels.

Among failure responders patients, there were 93.3\% had (BCR-ABL transcripts $>1 \%$ ). $15(50 \%$ ) out of them had a failure hematological response (FHR) and failure molecular response (FMR), 13(43.3\%) had only FMR and there were only $2(6.7 \%)$ with BCR-ABL levels between $(>0.1-1 \%)$ had FHR.

By the flow cytometry analysis of CD4+CD25+ T cells and PD1 expression among these cells in CML patients treated with imatinib therapy (Fig. 2). The (mean $\pm \mathrm{SD}$ ) of $\mathrm{CD} 4+\mathrm{T}$ cells percentage for the control, optimal and failure response patients were $(37.70 \pm 7.96 \%$, $38.39 \pm 8.55 \%$ and $35.86 \pm 9.55 \%$, respectively) with $(\mathrm{P}=0.5)$. Among these $\mathrm{CD} 4+\mathrm{T}$ cells the median of $\mathrm{CD} 4+\mathrm{CD} 25+$ Tregs percentage for the control was 8.8 , range (4.8-16.2), for optimal response 16.6, range (5.250.2 ) and for failure response patients was 71.7 , range (52.0-92.8). There was no significant difference between control and optimal groups with $(\mathrm{P}=0.481)$, while there was a significant increase in percentage of CD4+CD25+ Tregs in failure groups compared to the control and 
optimal groups $(\mathrm{P}=<0.001)$ for both as shown in (Table 1; Fig. 3).

Regarding PD1expression, data demonstrate no significant differences $(\mathrm{P}=0.125)$ in $\mathrm{PD} 1+$ among
CD4+CD25+ $\mathrm{T}$ cells, although data fail to reach significancy but there was a higher expression in PD1 percentage in failure groups compared to optimal and control groups (Table 1).

Table 1: Assessment of CD4+CD25+ T cells percentage among different CML patients and control group.

\begin{tabular}{|l|c|c|c|c|}
\hline Percentage (\%) & Control (30) & Optimal (30) & Failure (30) & P value \\
\hline${ }^{*} \mathrm{CD} 4+$ & $37.70 \pm 7.96$ & $38.39 \pm 8.55$ & $35.86 \pm 9.55$ & 0.511 \\
\hline${ }^{*} \mathrm{CD}^{+}{ }^{\mathrm{CD}} 25^{+}$in CD4 ${ }^{+}$T cells (range) & $8.8(4.8-16.2)$ & $16.6(5.2-50.2)$ & $71.7(52.0-92.8)$ & $<0.001$ \\
\hline${ }^{*} \mathrm{PD} 1+$ in CD4+ CD25+ cells & $65.30 \pm 18.88$ & $61.70 \pm 18.96$ & $71.60 \pm 18.56$ & 0.125 \\
\hline
\end{tabular}

*Presented using their mean \pm standard deviation, \# Presented using their median (interquartile range)

A) Control

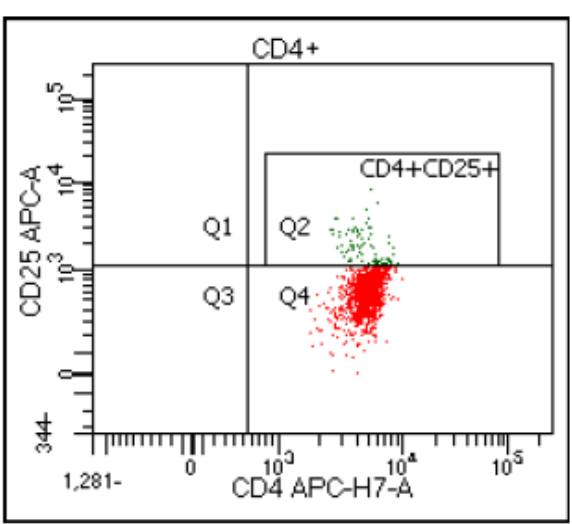

B)

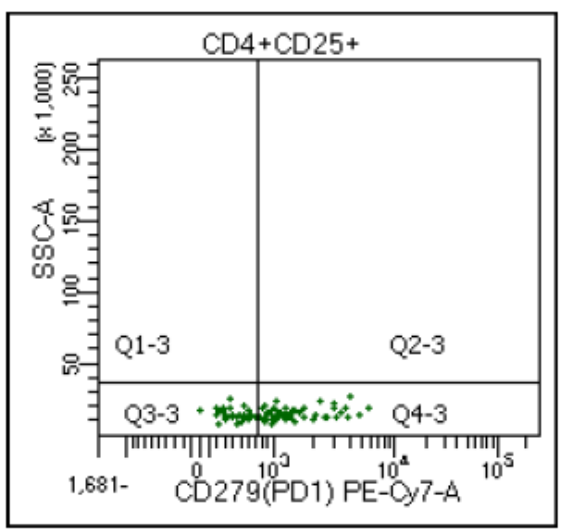

Optimal
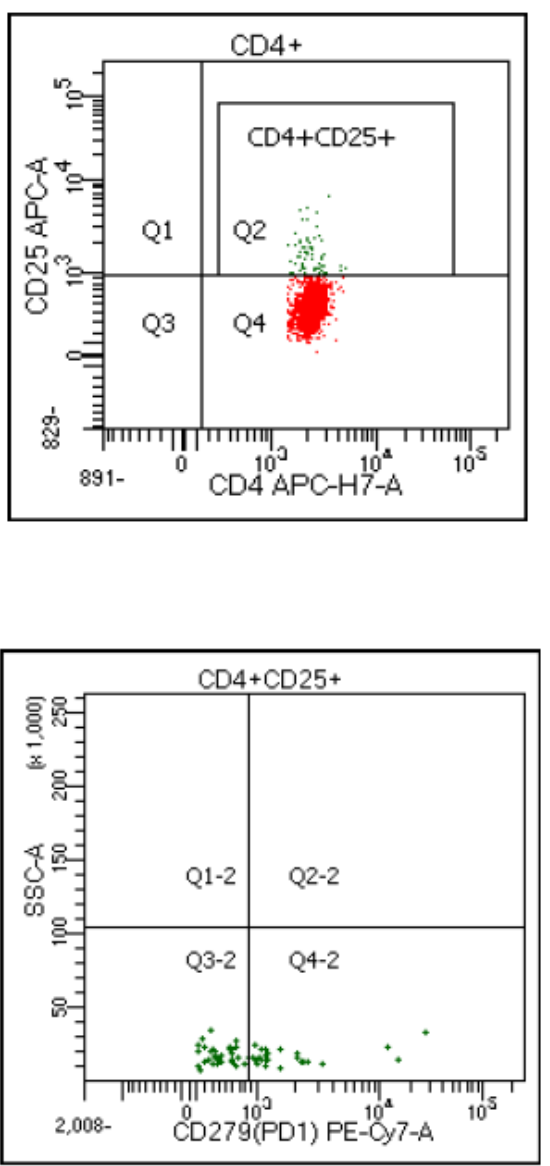

Failure

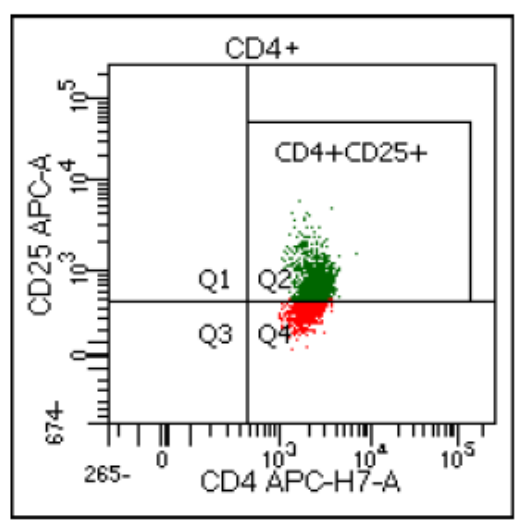

Figure 2: Flow cytometric analysis among different CML groups and control group. 
A) Gate for CD4+CD25+Tregs in all CD4+ T cells B) Gate for PD1 expression in CD4+CD25+ Tregs.

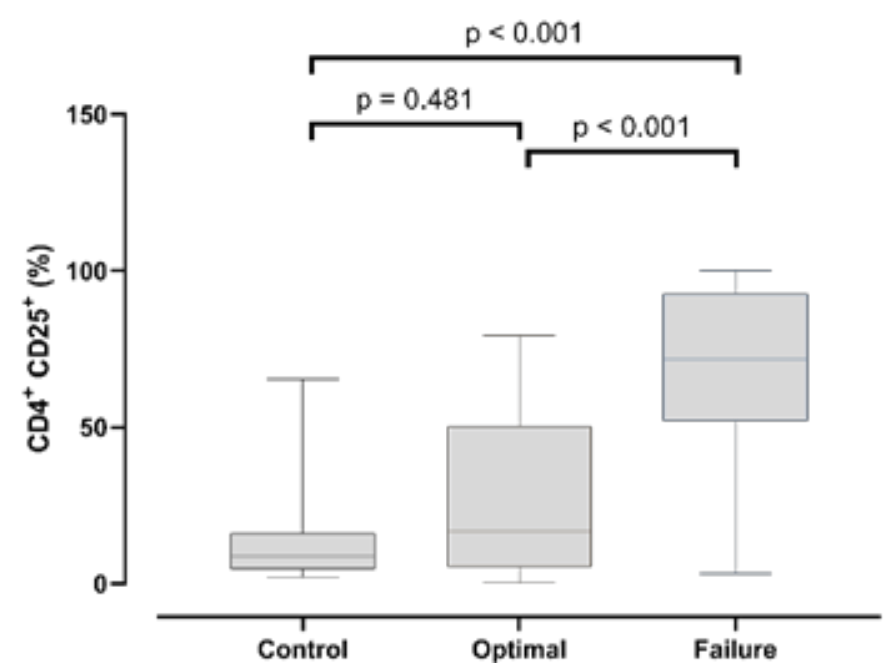

Figure 3: Percentage of $\mathrm{CD4}^{+} \mathrm{CD25}^{+} \mathrm{T}$ cells among different $\mathrm{CML}$ groups and control group.

Receiver operating characteristic (ROC) curve analysis was applied in order to determine a cutoff value of CD4+CD25+ Tregs by comparing their percentage among studied groups. Data failed to discriminate between optimal response and healthy control as shown in (Table 2). However, a cutoff value at ( $>33.7 \%$ ) was highly significant with high sensitivity and specificity that can be utilized to discriminate failure from optimal responders as shown in (Table 3 ).

Table 2: The Roc analysis for CD4+CD25+ Tregs percentage in studied groups

\begin{tabular}{|l|c|c|c|c|c|c|c|}
\hline Groups/\%CD4+CD25+ & AUC & Explanation & $\mathbf{9 5 \% C I ~ o f ~ A U C ~}$ & $\boldsymbol{P}$ value & Cut off \% & SN & SP \\
\hline Optimal vs Healthy & 0.619 & Poor & 0.484 to 0.741 & 0.115 & - & - & - \\
\hline Optimal vs Failure & 0.864 & Good & 0.751 to 0.939 & $<0.001$ & $>33.7$ & 96.7 & 70 \\
\hline
\end{tabular}

AUC: area under the curve, CI: confidence interval, SN: sensitivity, SP: specificity

\section{Discussion}

Imatinib mesylate has an influence on the immune cells that can be both suppressive or stimulating ${ }^{(10)}$. There is a clear discordance in immune response among CML patients, exemplified by the presence of many patients losing their response during the TKI treatment, whereas some others can develop deep molecular response, making the choice of stopping TKI treatment feasible for those patients ${ }^{(11)}$.

In this study, we include 60 patients of CML with mean age $46.8 \pm 13.7$, range (18-75) years old, the male:female ratio $1.14: 1$ which is comparable to ELN 2020 review as a younger age distribution among Asian population was $<50$ years old ${ }^{(12)}$. This study showed that the peripheral blood indices were lower in hemoglobin level and higher in WBC counts among failure group compared to the control and the optimal responders groups. This is related to bone marrow suppression by an increased leukemic clone cells due to loss of TKI response and transformation of CML patient to an advanced stage. The optimal responders CML patients also showed a lower hemoglobin level than the control group, which might be linked to the effect of long usage of imatinib treatment as the mean duration of treatment in our cohort where $73.7 \pm 37.6$ months, this is agree with Moura et al., ${ }^{(13)}$. The median platelets count among all studied groups was within normal range and this doesn't exclude presence of different phases of CML in this study.

Among CML patients with failure molecular response to imatinib therapy, there was significant $(\mathrm{P}=$ 
$<0.001)$ higher median of CD4+CD25+Tregs $(71.7 \%)$ in comparison to both optimal responders patients (16.6\%) and control group (8.8\%), without significant differences between the control group and the optimal responders patients. The presences of Tregs with high median level among non-responders CML patients, may reflect the suppression degree of immune effector cells response to the increased leukemic clone cells, and this may be through contribution of Tregs to inhibit the immune response to the disease activity. Conversely, the optimal responder CML patients showed much lower median level than the patients with failure response to therapy, with non-significant difference with the control group. This might be due to that the leukemic cell burden among the responders patients to imatinib therapy were much lower, indicating the efficacy of treatment that directly or indirectly reduced the Tregs counts and reconstitute the immune function. Our results are in line with Larmonier et al., ${ }^{(10)}$ who found through in vivo and in vitro investigations that imatinib therapy decreased Treg frequency and impaired their immune suppressive function. Recent study showed that imatinib treated CML patients in complete molecular remission exhibited selective depletion of effector Treg cells and significant increase in effector memory CD8 $+\mathrm{T}$ cells while non-complete molecular remission did not ${ }^{(14)}$.

Moreover, studies showed CD4+CD25+ Treg cells depletion reinstated an efficient anti-tumor immune response leading to complete tumor regression (15). The possibility that the function of Tregs may also influence patients with cancer has been suggested by previous studies on CLL (16) and lymphoma malignancies ${ }^{(17)}$. Therefore, in relation to CML disease, the immunosuppressive properties of regulatory $\mathrm{T}$ cells seem to be particularly important in disease control status.

Our study established a cutoff value of CD4+CD25+ $\mathrm{T}$ cells at $\mathbf{3 3 . 7 \%}$ which might be a good biomarker to distinguish between failure and optimal responders to TKI treatment.

In present study, the data analysis didn't show noticeable difference in mean levels of PD1 expression on Tregs among the studied groups, despite the higher mean level in failure responders CML patients. This may relate to the extent of PD-1 mediated inhibition depending on the strength of the TCR signal, where as PD-L1 expression on non-lymphoid tissues and its potential interaction with PD1 may subsequently determine the extent of immune responses ${ }^{(18)}$. Park et al., (19) suggested that PD1 expression in Tregs and the PD1/ PDL1 interplay is responsible for T cells exhaustion and can inhibit their proliferation and cytokines releasing.

\section{Conclusion}

CML disease undergoing TKI therapy is immune responsive disease, this response determined by the degree and type of CML molecular response, which may play a vital role in controlling the disease activity in advanced stage and deeper molecular response in patient who achieved MMoR. Our observation highlights the importance of Treg function and its interaction with PD1 among CML patients. Furthermore, our findings might turn the focus of the new therapies towards targeting these escape mechanisms.

\section{Conflict of Interest: None}

\section{Funding: Self}

Ethical Clearance: Not required

\section{Références}

1. Hughes A, Clarson J, Tang C, Vidovic L, White DL, Hughes TP, Yong AS. CML patients with deep molecular responses to TKI have restored immune effectors and decreased PD- 1 and immune suppressors. Blood, The Journal of the American Society of Hematology. 2017 Mar 2;129(9):116676.

2. Tarafdar A, Hopcroft LE, Gallipoli P, Pellicano F, Cassels J, Hair A, Korfi K, Jørgensen HG, Vetrie D, Holyoake TL, Michie AM. CML cells actively evade host immune surveillance through cytokinemediated downregulation of MHC-II expression. Blood, The Journal of the American Society of Hematology. 2017 Jan 12;129(2):199-208.

3. Hochhaus A, Saussele S, Rosti G, Mahon FX, Janssen JJ, Hjorth-Hansen H, Richter J, Buske C, ESMO Guidelines Committee. Chronic myeloid leukaemia: ESMO Clinical Practice Guidelines for diagnosis, treatment and follow-up. Annals of Oncology. 2017 Jul 1;28(suppl_4):iv41-51.

4. Brück O, Blom S, Dufva O, Turkki R, Chheda H, Ribeiro A, Kovanen P, Aittokallio T, Koskenvesa P, Kallioniemi O, Porkka K. Immune cell contexture in the bone marrow tumor microenvironment impacts therapy response in CML. Leukemia. 2018 Jul;32(7):1643-56. 
5. Wu CP, Qing X, Wu CY, Zhu H, Zhou HY. Immunophenotype and increased presence of $\mathrm{CD} 4+\mathrm{CD} 25+$ regulatory $\mathrm{T}$ cells in patients with acute lymphoblastic leukemia. Oncology Letters. 2012 Feb 1;3(2):421-4.

6. Tanaka A, Sakaguchi S. Regulatory $\mathrm{T}$ cells in cancer immunotherapy. Cell research. 2017 Jan;27(1):109-18.

7. Cai J, Wang D, Zhang G, Guo X. The role of PD-1/PD-L1 Axis in Treg development and function: implications for Cancer immunotherapy. OncoTargets and therapy. 2019;12:8437.

8. Hughes A, Yong AS. Immune effector recovery in chronic myeloid leukemia and treatment-free remission. Frontiers in immunology. $2017 \mathrm{Apr}$ 24;8:469.

9. Baccarani M, Deininger MW, Rosti G, Hochhaus A, Soverini S, Apperley JF, Cervantes F, Clark RE, Cortes JE, Guilhot F, Hjorth-Hansen $\mathrm{H}$. European LeukemiaNet recommendations for the management of chronic myeloid leukemia: 2013. Blood. 2013 Aug 8;122(6):872-84.

10. Larmonier $\mathrm{N}$, Janikashvili $\mathrm{N}$, LaCasse CJ, Larmonier CB, Cantrell J, Situ E, Lundeen T, Bonnotte B, Katsanis E. Imatinib mesylate inhibits $\mathrm{CD} 4+\mathrm{CD} 25+$ regulatory $\mathrm{T}$ cell activity and enhances active immunotherapy against BCRABL - tumors. The Journal of Immunology. 2008 Nov 15;181(10):6955-63.

11. Mahon FX, Etienne G. Deep molecular response in chronic myeloid leukemia: the new goal of therapy?. Clinical Cancer Research. 2014 Jan 15;20(2):310-22.

12. Hochhaus A, Baccarani M, Silver RT, Schiffer C, Apperley JF, Cervantes F, Clark RE, Cortes JE, Deininger MW, Guilhot F, Hjorth-Hansen H. European LeukemiaNet 2020 recommendations for treating chronic myeloid leukemia. Leukemia. 2020 Mar 3:1-9.

13. Moura MS, Benevides TC, Delamain MT, Duarte GO, Percout PO, Dias MA, Zulli R, Souza CA, Lorand-Metze I, Pagnano KB. Evaluation of

anemia after long-term treatment with imatinib in chronic myeloid leukemia patients in chronic phase. Hematology, transfusion and cell therapy. 2019 Dec;41(4):329-34.

14. Tanaka A, Nishikawa H, Noguchi S, Sugiyama D, Morikawa H, Takeuchi Y, Ha D, Shigeta N, Kitawaki T, Maeda Y, Saito T. Tyrosine kinase inhibitor imatinib augments tumor immunity by depleting effector regulatory T cells. The Journal of experimental medicine. $2020 \mathrm{Feb} 3 ; 217(2)$.

15. Beyer M, Kochanek M, Giese T, Endl E, Weihrauch MR, Knolle PA, Classen S, Schultze JL. In vivo peripheral expansion of naive CD4+ CD25high FoxP3 + regulatory $\mathrm{T}$ cells in patients with multiple myeloma. Blood. 2006 May 15;107(10):3940-9.

16. Beyer M, Kochanek M, Darabi K, Popov A, Jensen M, Endl E, Knolle PA, Thomas RK, von Bergwelt-Baildon M, Debey S, Hallek M. Reduced frequencies and suppressive function of $\mathrm{CD} 4+\mathrm{CD} 25$ hi regulatory $\mathrm{T}$ cells in patients with chronic lymphocytic leukemia after therapy with fludarabine. Blood. 2005 Sep 15;106(6):2018-25.

17. Álvaro T, Lejeune M, Salvadó MT, Bosch R, García JF, Jaén J, Banham AH, Roncador G, Montalbán C, Piris MA. Outcome in Hodgkin's lymphoma can be predicted from the presence of accompanying cytotoxic and regulatory $\mathrm{T}$ cells. Clinical Cancer Research. 2005 Feb 15;11(4):1467-73.

18. Freeman GJ, Long AJ, Iwai Y, Bourque K, Chernova T, Nishimura H, Fitz LJ, Malenkovich N, Okazaki T, Byrne MC, Horton HF. Engagement of the PD-1 immunoinhibitory receptor by a novel B7 family member leads to negative regulation of lymphocyte activation. The Journal of experimental medicine. 2000 Oct 2;192(7):1027-34.

19. Park HJ, Park JS, Jeong YH, Son J, Ban YH, Lee BH, Chen L, Chang J, Chung DH, Choi I, Ha SJ. PD-1 upregulated on regulatory $\mathrm{T}$ cells during chronic virus infection enhances the suppression of $\mathrm{CD} 8+\mathrm{T}$ cell immune response via the interaction with PD-L1 expressed on CD8+ T cells. The Journal of Immunology. 2015 Jun 15;194(12):5801-11. 